\title{
THE CHALLENGE OF ANTIPSYCHOTIC-INDUCED TARDIVE DYSKINESIA MANAGEMENT IN SCHIZOPHRENIC PATIENTS
}

O.Nombora ${ }^{1}$, A. Samico ${ }^{1}$, A. Venâncio ${ }^{1}$

${ }^{1}$ Psychiatry and Mental Health Service, Vila Nova de Gaia/Espinho Hospital Center, Portugal Corresponding Author: odete.nombora@gmail.com

\section{BACKGROUND}

Tardive dyskinesia (TD) is a disabling chronic movement disorder, characterized by involuntary hyperkinetic movements that is often persistent, irreversible, and has a low tendency of remission. It typically appears after at least 3 months or longer of treatment with dopamine receptor blocking agents, mostly antipsychotics $[2,3,8,6,7,9,10]$. Patients with moderate to severe TD generally have a significant impairment in their quality of life and it can also lead to poor treatment adherence. Many strategies have been created in order to manage TD, but the majority of patients still does not receive early diagnosis, which leads to a delay or inaccurate treatment and poor outcome ${ }^{[3,10]}$. Therefore prompt recognition and implementation of effective treatment regimens and long-term follow up of patients with TD is essential.

\section{OBJECTIVE}

Through a clinical case presentation, we aim to provide an overview of TD epidemiology and treatment recommendations available.

\section{METHODS}

We present a clinical case and a qualitative review about epidemiology and treatment of TD. We made a PubMed search using the terms "tardive dyskinesia", "antipsychotic induced tardive dyskinesia", "tardive dyskinesia in schizophrenia", "tardive dyskinesia management", "tardive dyskinesia epidemiology".

\section{RESULTS AND DISCUSSION}

\section{CLINICAL CASE}

54 year-old woman, divorced, lives with both her sons. Retired, worked as a taxi driver. Irrelevant surgical and medical background.

About six years ago she was compulsively hospitalized due to a psychotic decompensation, diagnosed with Paranoid Schizophrenia. Initially treated with Olanzapine $20 \mathrm{mg} /$ day and posteriorly with Risperidone $9 \mathrm{mg} /$ day, without therapeutic response, so it was decided to introduce Haloperidol. She went home with haloperidol decanoate $100 \mathrm{mg}$ (28/28 days), haloperidol $10 \mathrm{mg} /$ day, Biperiden $8 \mathrm{mg} /$ day and Lorazepam $3 \mathrm{mg} /$ day.

In 2017, she began to present clinical manifestations compatible with the diagnosis of TD - with complex, repetitive and stereotypical movements of mastication, suction and protrusion of the tongue and choreic movements with dystonic postures of the limbs. The movements were exacerbated by distraction and stress, suppressed voluntarily and disappeared with sleep. No improvement seen with reduction and suspension of oral haloperidol. Tetrabenazine up to $75 \mathrm{mg} /$ day was introduced without response and the dose could not be increased due to side effects (hypotension and drowsiness). She starts doing Amantadine 100mg/day and then Gingko-biloba 160mg/day, without response. It was then decided to switch the antipsychotic to Clozapine, with symptoms improvement. She is currently doing Clozapine at $250 \mathrm{mg} / \mathrm{day}$, with clinical stabilization.

\section{EPIDEMIOLOGY}

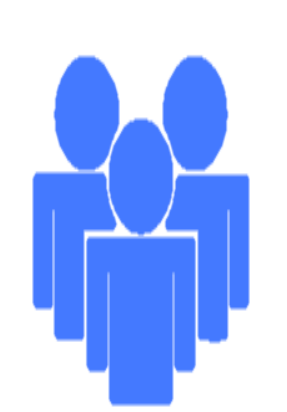

Up to $30 \%$ of patients using antipsychotic drugs develop TD. Prevalence may be higher in selected populations ${ }^{[3,7,9,10]}$.The annual incidence rate is estimated at $\mathbf{5 \%}$ with a cumulative 5 -year incidence of $\mathbf{2 5 \%}{ }^{[3,10]}$. TD can be reversible in approximately $50 \%$ of patients ${ }^{[9,10]}$.

MANAGEMENT

- Recognition and characterization of TD manifestations - Monitoring every 3-12 months depending on patient risk factors and antipsychotic $[9,10]$

Tetrabenazine has been approved for TD but its potential side effects minimize its use. $[1,2,3,9,10]$

\section{CONCLUSIONS}

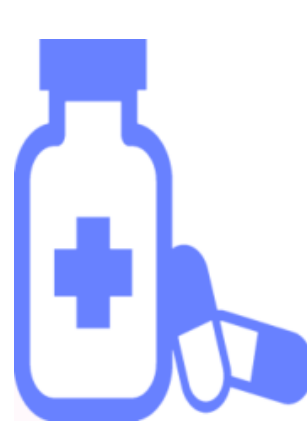

Incidence is higher with first generation antipsychotics (FGA) comparing to second generation antipsychotics (SGA). But all antipsychotic groups carry a risk for TD. $[3,5,6,7,10]$

If moderate to severe

\begin{tabular}{l|l} 
If mild TD & - Discontinuation of \\
anticholinergic \\
agents as they can \\
symptoms \\
worsen \\
symptoms \\
- Antipsychotic \\
dose reduction or \\
tapered withdrawal \\
when possible \\
{$[3,5,6,7,8,10]$}
\end{tabular}

Deep brain stimulation (Level C), might be considered as a treatment for intractable TD symptoms

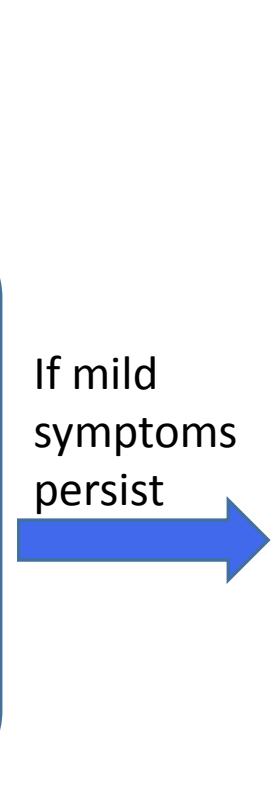
FGA to a SGA with lower affinity to D2 receptors can be considered $[3,5,6,7,8,10]$
Add Specific antidyskinetic drugs (if available), Vesicular monoamine transporters 2 (VMAT2) inhibitors (Level A): Valbenazine or Deutetrabenazine.

No response

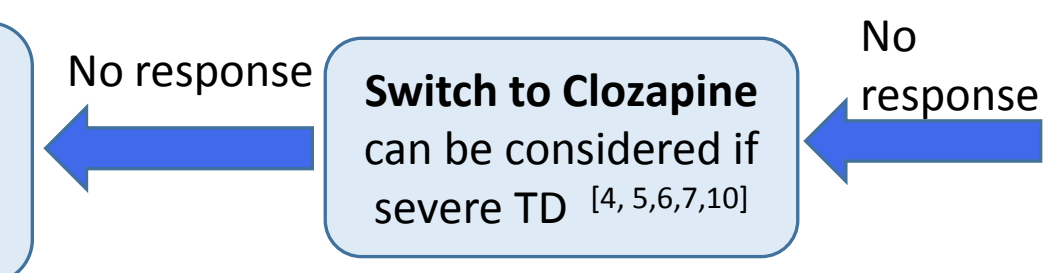

Chronic use of antipsychotics (particularly high dose), elderly ( $>60$ years), female sex, organic brain disease and history of previous drug-induced movement disorder are the main risk factors $[3,4,7,9,10]$.

TD management is challenging in schizophrenic patients. Despite the use of SGA it remains relatively common. Switch to Clozapine is still the main approach to consider in patients with severe TD symptoms in most countries where VMAT2 inhibitors are not available. Careful clinical assessment is important to recognize TD for its prompt management and further research in this field is needed.

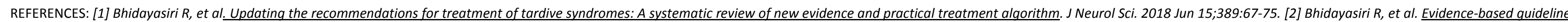

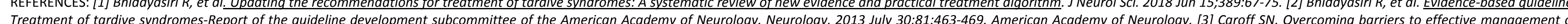

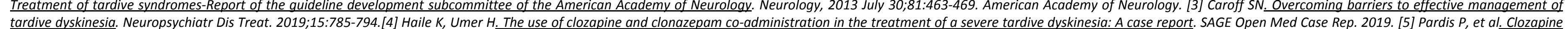

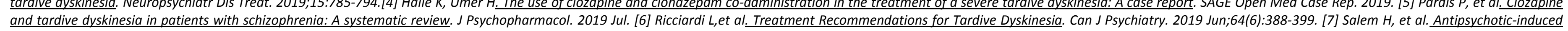

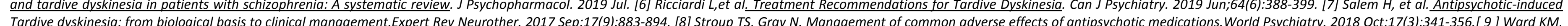

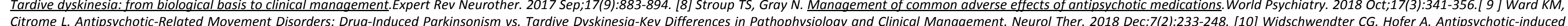

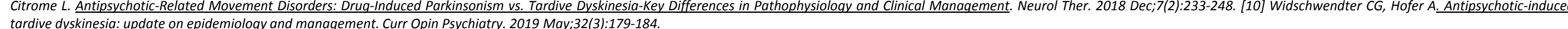

